\title{
Mouillabilité et interactions solide-liquide dans l'encrassement de divers matériaux par du lactosérum et du lait
}

\author{
Monique DUPEYRAT *, J.-P. LABBÉ **, Françoise MICHEL ***, \\ Françoise BILLOUDET *, G. DAUFIN *** \\ * Université Paris VI, Laboratoire de Chimie Physique (UA 176) \\ 11, rue Pierre-et-Marie-Curie, 75231 Paris Cedex 05, France \\ ** Ecole Nationale Supérieure de Chimie, Laboratoire de Corrosion \\ 11, rue Pierre-et-Marie-Curie, 75231 Paris Cedex 05, France \\ *** INRA, Laboratoire de Recherches de Technologie laitière \\ 65, rue de Saint-Brieuc, 35042 Rennes Cedex, France
}

\section{Résumé}

Les interactions solide-liquide apparaissant au cours de l'encrassement de divers matériaux par des fluides laitiers (lactosérum, lait, solutions modèles), sont étudiées à partir de mesures de masses de dépôts, de mouillabilité à l'eau de surfaces nues et encrassées et d'analyse par spectroscopie infrarouge. L'encrassement est réalisé dans des conditions standardisées $\left(72{ }^{\circ} \mathrm{C}\right.$; pas de gradient de température) à l'aide de deux dispositifs d'encrassement. L'influence de la nature du matériau solide (acier inoxydable, Téflon, verre, ...), de l'état de surface et du procédé de nettoyage (base puis acide : type "laiterie "; mélange sulfochromique ; solvants organiques des matières grasses) a été examinée. Il en résulte que ces interactions se font au travers du film d'eau qui mouille systématiquement la paroi solide et tend à uniformiser la nature de l'encrassement. On ne peut certes pas exclure l'intervention d'interactions physiques ou chimiques locales dépendant de la nature du matériau solide dans les toutes premières étapes de l'encrassement. Mais l'effet de la paroi s'estompe très vite et la croissance du dépôt se fait par interaction des composants du fluide encrassant avec les produits qui en sont issus par l'intermédiaire du solvant (eau), si bien que la nature et la quantité de dépôt sont pratiquement toujours les mêmes quels que soient le matériau et l'état de surface de ce dernier.

Mots clés : Encrassement - Lait - Lactosérum - Mouillabilité - Infrarouge - Matériau solide - Etat de surface.

\section{Summary}

Wettability and solid-liquid interactions during fouling of several materials by whey and milk

The solid-liquid interactions occuring during fouling of different materials by dairy products (whey, milk, model solutions) were studied from deposit masses, wettability of bare and fouled surfaces and Infra-Red spectroscopy. Fouling experiments were carried out 
in standardized conditions ( $72{ }^{\circ} \mathrm{C}$; no temperature gradient) using two fouling laboratory devices. The influence of solid material nature (stainless steel, Teflon, glass,...), surface finish and cleaning process (alkaline-acid: dairy type, sulphochromic mixture, organic solvents for lipids) were studied. It is concluded that the interactions occur through a water film which systematically wets the solid wall and tends to give a similar nature to the deposits. Of course, local chemical or physical interactions depending on solid material nature cannot be excluded at the very beginning of fouling. But after these first steps, the material nature becomes unimportant, as the deposit growth mainly depends on interactions between the fouling fluid and solid substances built-up through the solvent (water). The nature and amount of deposit are therefore almost the same whatever the material and the surface finish.

Key words : Fouling - Milk - Whey - Wettability - Infra-Red - Solid material - Surface finish.

\section{Introduction}

La mise au point bibliographique de LunD et SANDU (1981) souligne que les recherches sur les interactions matériaux solides-produits alimentaires n'ont pas suivi le développement de celles qui ont été entreprises dans le domaine de l'encrassement rencontré dans d'autres secteurs biologiques («fouling » marin ; plaque dentaire ; biocompatibilité ; ...) (BAIER, 1981). Pourtant, il s'agit là d'un domaine d'investigation nouveau qui prend en compte l'interface matériau-produit alimentaire. En effet, comme dans le « fouling », des interactions entre la surface solide et les constituants biologiques des aliments sont en compétition avec les interactions intervenant entre ces constituants dans la masse de l'aliment. Quelques équipes étrangères ont commencé à aborder des études sur ces interactions dans le cas de matériaux métalliques en contact avec du lait ou ses constituants : HegG et LarsSon (1981) et Ivarsson et al. (1985) par ellipsométrie, électrochimie et mesures d'angles de contact ; IvarsSON et al. (1981) essentiellement par ellipsométrie; BAIER (1981) par InfraRouge et mesures d'angles de contact. De nombreuses données cinétiques et analytiques sur l'encrassement d'une surface d'échange de chaleur en acier inoxydable $304 \mathrm{~L}$ à l'état poli mécanique (granulométrie $12 \mu \mathrm{m}$ ) ont été récemment acquises pour des fluides laitiers (lait, lactosérum) (DAUFIN et al., 1987). L'encrassement dépend évidemment des interactions entre le matériau et la solution aqueuse. L'énergie d'interaction peut être exprimée, au signe près, à partir de l'énergie d'adhésion par aire unité, $\mathrm{W}_{\mathrm{A}}$ définie par Dupré (1869). On montre classiquement (DUPEYRAT, 1983) que :

$$
\mathrm{W}_{\mathrm{A}}=\gamma_{\mathrm{L}}(1+\operatorname{Cos} \theta) \text {. }
$$

$\gamma_{\mathrm{L}}$ et $\theta$ sont respectivement la tension superficielle du liquide et l'angle de contact entre le liquide et le solide au point de raccordement des deux phases, pris dans le liquide. $\gamma_{\mathrm{L}}$ est mesurable classiquement à l'aide d'un tensiomètre. $\theta$ peut l'être à l'aide d'un goniomètre, mais l'angle est difficile à apprécier au voisinage de 0 et de $180^{\circ}$. Par contre, $\mathrm{W}_{\mathrm{A}}$ peut être évalué à partir du travail de mouillage $\left(\mathrm{W}_{\mathrm{m}}\right)$ par aire unité, selon la relation $\mathrm{W}_{\mathrm{A}}=\gamma_{\mathrm{L}}-\mathrm{W}_{\mathrm{m}}$ ce qui conduit à $\operatorname{Cos} \theta=-\mathrm{W}_{\mathrm{m}} / \gamma_{\mathrm{L}}$ (DUPEYRAT, 1983).

Par conséquent, notre premier objectif a été d'établir des corrélations entre, d'une part les caractéristiques de mouillabilité des surfaces avant ou 
après encrassement et, d'autre part, la composition et les propriétés structurales des dépôts obtenus à partir de lactosérum et de lait (déduites de la spectrométrie infrarouge). Evaluer comment les masses d'encrassement sont affectées par des modifications de la surface (différentes finitions de l'acier inoxydable ; différents matériaux) a constitué notre second objectif.

Cette étude cherche donc à apporter quelques éléments nouveaux concernant le mécanisme d'encrassement d'une part, et des idées pour lutter pratiquement contre l'encrassement d'autre part.

\section{Matériel et méthodes}

\section{A. Matériel}

\section{Dispositifs et conditions d'encrassement}

L'encrassement est réalisé dans une installation de laboratoire comprenant un préchauffeur tubulaire et un dispositif d'encrassement. Ce dernier est constitué, soit d'une maquette d'échangeur de chaleur, décrite de façon détaillée par Roignant et al. (1983), soit d'une cellule donnant la possibilité de réaliser un dépôt sur les deux faces du matériau solide. La maquette a l'avantage de permettre une bonne maîtrise des conditions hydrodynamiques et thermiques dans lesquelles l'encrassement s'effectue seulement sur une partie de l'aire $(2 \times 4 \mathrm{~cm})$ d'une seule face du matériau $(3 \times 5 \mathrm{~cm})$. Les dépôts correspondants sont utilisés pour la mesure de l'angle de contact (paragr. B.3.a). La cellule (fig. 1) est constituée d'une «boîte» parallélépipédique en Duralumin de dimensions intérieures $4 \times 2,8 \times 0,3 \mathrm{~cm}$, calorifugée par un

Fig. 1

Schéma de la cellule: $C$ : corps; $E, S$ : entrée et sortie du fluide; $L$ : lame étudiée épaisseur 0,6 $\mathrm{mm}$.

Scheme of the fouling cell for wettability measurements: $C$ : body; $E, S$ : fluid inlet and outlet; $L$ : plate studied - width 0.6 $\mathrm{mm}$.

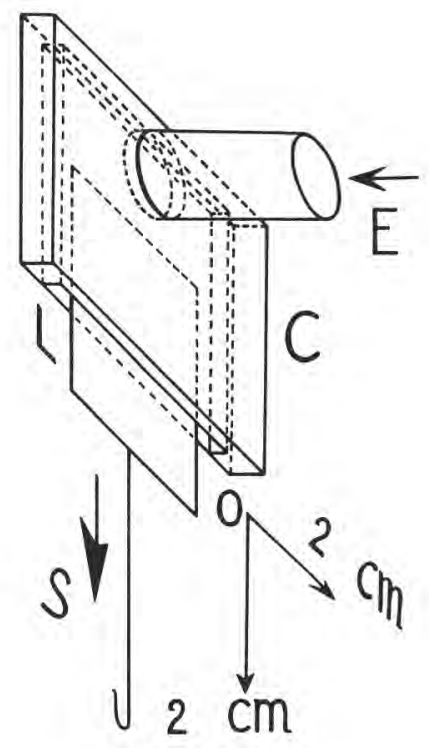


revêtement en polystyrène expansé, ouverte à la partie inférieure. Elle porte latéralement une tubulure en $\mathrm{T}$ de diamètre $0,8 \mathrm{~cm}$, servant à l'amenée des fluides. La lame à encrasser est introduite à la partie inférieure de la boîte. Le bord inférieur de la tubulure est situé à $0,3 \mathrm{~cm}$ de l'extrémité supérieure de la lame de sorte que les fluides arrivent sur les deux faces de la lame. Sur la partie inférieure de cette lame, l'écoulement se fait au contact de l'air, ce qui peut provoquer une adsorption de certains composants, les lipides en particulier, à l'interface air/phase aqueuse, susceptible de modifier les conditions de dépôt sur la lame, comme dans le cas d'une bulle. Cette région de la lame n'est donc pas utilisée pour les mesures. La géométrie de la cellule diminue considérablement la formation de bulles au cours de l'écoulement. Cependant les deux coins supérieurs de la lame sont souvent mal encrassés. Cette portion ne sera donc pas retenue pour l'interprétation des mesures.

Les conditions hydrodynamiques et thermiques sont voisines pour les deux dispositifs. Suivant qu'il s'agit de la maquette d'échange de chaleur ou de la cellule, respectivement, les temps de séjour moyens des fluides dans le préchauffeur sont de 28 ou 14 secondes. La vitesse d'écoulement le long de la surface étudiée est 0,096 ou $0,11 \mathrm{~m} \cdot \mathrm{s}^{-1}$. Comme l'ont montré Daufin et al. (1987), la masse d'encrassement augmente fortement à partir de $60-70^{\circ} \mathrm{C}$. Les essais sont donc réalisés à $72{ }^{\circ} \mathrm{C} \pm 2$, la paroi et le fluide étant à la même température. Ces conditions seront qualifiées de standard dans la suite. La durée des essais est fixée à 10 ou 20 minutes selon le cas.

\section{Fluides}

Les fluides utilisés sont décrits en détail (mode de préparation ; composition chimique, ...) dans un travail récent (DaUfin et al., 1985).

Il s'agit de deux fluides laitiers industriels conservés sous forme de poudre: lactosérum doux de pâte cuite reconstitué à raison de $63,2 \mathrm{~g}$ de matière sèche par $\mathrm{kg}$ de produit ; lait écrémé reconstitué à un taux de matière sèche de $90 \mathrm{~g} \cdot \mathrm{kg}^{-1}$. En outre, quelques solutions modèles soigneusement choisies permettent une comparaison du rôle des différents constituants de ces fluides : solution de «phosphate et calcium» (on désignera ainsi la solution obtenue à partir d'hydrogénophosphate de potassium et de chlorure de calcium ajustée du point de vue de la force ionique et du $\mathrm{pH}$ ); ultrafiltrat synthétique ; lactosérum à calcium complexé par du citrate. Pour tous les fluides, le $\mathrm{pH}\left(\right.$ à $20^{\circ} \mathrm{C}$ ) était de 6,6 (pH du lait).

\section{Matériaux}

a) Matériaux et prétraitements de surface

Dans l'ensemble de notre travail, l'acier inoxydable $304 \mathrm{~L}$ poli mécaniquement au papier abrasif de granulométrie $12 \mu \mathrm{m}$ constituait le matériau et l'état de surface de référence. Dans la recherche du rôle des caractéristiques superficielles des matériaux, l'acier inoxydable $304 \mathrm{~L}$ était utilisé aussi à l'état brut de laminage "glacé » (BLG) (Usinor) ou poli électrolytiquement (Calomo, Suède). En outre, à titre de comparaison, divers essais ont été effectués sur des matériaux très différents de ceux que l'on utilise dans les échangeurs 
industriels : verre Pyrex (code 7740, Corning); Téflon (PTFE) et polyéthylène (PE) massifs; alliage d'aluminium ASG 6080 oxydé anodiquement ( $20 \mu \mathrm{m})$ puis colmaté à l'eau (Péchiney); films minces de résines en silicone noire (M 18, Applifrance) ou fluorée (P30 + F40, Applifrance) appliqués sur des tôles en aluminium de $1 \mathrm{~mm}$ d'épaisseur.

Enfin, la surface de l'acier inoxydable $304 \mathrm{~L}$ polie $(12 \mu \mathrm{m})$ peut être modifiée par un prétraitement consistant à l'encrasser à l'aide de lactosérum ou d'ultrafiltrat synthétique circulant dans la maquette dans les conditions énoncées précédemment $\left(72^{\circ} \mathrm{C} ; \Delta \mathrm{T}=0^{\circ} \mathrm{C} ; 20 \mathrm{mn}\right)$.

\section{b) Procédés de nettoyage}

Des essais préliminaires ayant montré une influence du nettoyage de la surface sur l'encrassement, plusieurs procédés de nettoyage ont été utilisés.

- Nettoyage au mélange sulfochromique (dissolution de $\mathrm{K}_{2} \mathrm{Cr}_{2} \mathrm{O}_{7}$ cristallisé dans $\mathrm{H}_{2} \mathrm{SO}_{4}$ concentré jusqu'à ce que du papier «Joseph » soit détruit en moins de 30 secondes). La lame est immergée dans le mélange pendant une vingtaine de secondes puis rincée, d'abord avec un litre d'eau courante pendant 15 secondes, ensuite avec un litre d'eau distillée courante pendant 30 secondes (élimination de toute trace de protéines et de lipides).

- Nettoyage à l'aide de divers solvants des graisses par immersion dans $50 \mathrm{ml}$ de solvant (trichloréthylène, hexane ou éthanol) pendant une trentaine de minutes en agitant la lame une quinzaine de fois (élimination des lipides).

- Nettoyage modèle du nettoyage industriel appelé dans la suite « nettoyage laiterie » dont la séquence est la suivante: lavage sous un débit de $601 . \mathrm{h}^{-1}$ avec $\mathrm{NaOH}, 2 \%$ à $65^{\circ} \mathrm{C}$ pendant 10 minutes, rinçage à l'eau distillée pendant 5 minutes, lavage avec $\mathrm{HNO}_{3}, 1,3 \%$, à $70^{\circ} \mathrm{C}$ pendant 5 minutes puis rinçage à l'eau distillée (5 minutes).

- Nettoyage par immersion dans l'éthanol sous ultrasons $(80 \mathrm{kHz})$ pendant 2 minutes à température ambiante.

\section{Protocole expérimental}

Avant chaque essai, l'installation est nettoyée selon un des procédés mentionnés. Ensuite, l'échangeur ou la cellule est mis en équilibre hydrodynamique et thermique à l'aide d'eau distillée pendant une durée de l'ordre de 10 minutes, puis le fluide encrassant est injecté sans solution de continuité en tournant une vanne à trois voies. Après l'encrassement, l'eau distillée de rinçage est introduite sans solution de continuité, pendant 5 minutes.

\section{B. Méthodologie}

\section{Pesée des dépôts}

L'importance de l'encrassement est évaluée par pesage à l'aide de balances Mettler : AE 163 (seuil de détection $10 \mu \mathrm{g}$ ) ou M5 (seuil de détection $2 \mu \mathrm{g}$ ). Les masses indiquées sont la moyenne d'au moins trois essais d'encrassement répétés dans les mêmes conditions. Elles sont déterminées systématiquement à l'issue des essais en maquette et seulement dans quelques cas particuliers pour les essais en cellule. 


\section{Etude des spectres infrarouges des dépôts}

Le prélèvement des dépôts sur les surfaces encrassées est effectué par la méthode mise au point par LABBÉ (DAufin et al., 1985). Les spectres sont enregistrés à partir des micropastilles correspondantes avec deux spectromètres différents: Perkin Elmer dispersif de routine (modèle 457) ou Brucker à transformée de Fourier, type 45.

L'aspect des spectres donne des indications sur la nature des composés présents : lipides, protéines, phosphates, essentiellement (DAUfin et al., 1985). La mesure des rapports internes d'absorbance permet d'esquisser des compositions modèles des dépôts (protéines, phosphates, lipides) comparables les unes aux autres (DAUFIN et al., 1985).

\section{Mesures de mouillabilité des surfaces avant et après encrassement}

\section{a) Mesure de l'angle de contact à l'aide d'un goniomètre}

La méthode adoptée est celle proposée par ANDRADE et al. (1979) à la suite des travaux d'HAMILTON (1972). Elle est appliquée aux lames encrassées dans la maquette.

Une cuve en polystyrène cristal, contenant un portoir en verre, emplie d'eau distillée $\left(\gamma_{\mathrm{L}}=72,8 \mathrm{mN} \cdot \mathrm{m}^{-1}\right.$, à $\left.20^{\circ} \mathrm{C}\right)$, est posée sur un cavalier situé sur un banc optique. Après avoir essuyé plusieurs fois la surface de l'eau avec du papier "Joseph", on immerge l'échantillon dont la surface à caractériser fait face au fond de la cuve, en le posant sur le portoir. Une microseringue Hamilton $(\mathrm{v}=1 \mu \mathrm{l})$, montée sur un support à mouvements micrométriques croisés (horizontal et vertical), permet de former, dans le champ de l'oculaire $(\mathrm{G}=7)$ du microscope viseur équipé d'un objectif $(\mathrm{G}=10)$, une bulle d'air dans des conditions telles qu'elle repousse correctement le film d'eau présent sur la surface. L'angle mesuré est donc un angle « reculant». La plupart des échantillons étudiés contenant de l'eau, il est difficile, même avec des précautions, de "démouiller » totalement la surface au contact de la bulle d'air. Il en résulte une erreur de mesure notable pour des angles compris entre 0 et $20^{\circ}$ d'une part, 180 et $160^{\circ}$ d'autre part. Chaque valeur donnée est la moyenne d'au moins 6 déterminations effectuées sur 3 bulles formées sur l'échantillon considéré.

b) Mesure du travail de mouillage ou de démouillage par la méthode de la lame de Wilhelmy (1863)

La méthode consiste à mesurer la force nécessaire pour augmenter de façon contrôlée l'aire de la surface libre lors de l'immersion ou de l'émersion dans le liquide d'une lame mince rectangulaire du solide étudié. Au cours de ce travail, le liquide étudié est toujours l'eau. On montre (DUPEYRAT, 1983) qu'on mesure ainsi la tension superficielle si la lame est parfaitement mouillable par le liquide, le travail de mouillage ou de démouillage selon que l'on travaille à l'immersion ou à l'émersion, dans le cas contraire.

Pour cela, on accroche la lame à un capteur de micro-force (Schenk Q 11 ; portée $0,5 \mathrm{~N}$; incertitude $0,5 \%$ de la force mesurée) qui mesure la somme du poids apparent de cette lame et de la force liée au mouillage ou au 
démouillage qui s'exerce sur cette dernière au cours de l'immersion ou de l'émersion de la lame respectivement. En effet, on trace la courbe à l'immersion puis, après inversion du sens de déplacement relatif du solide par rapport à la surface libre du liquide et du déroulement du papier, la courbe à l'émersion. L'ensemble est appelé «cycle » de mouillage. Il s'ensuit que, si le mouillage est spontané - ce qui est le cas dans nos expériences - $W_{m}$ mesuré pendant une immersion est négatif et est représenté par le travail de démouillage correspondant, $\mathrm{W}_{\mathrm{dem}}\left(=-\mathrm{W}_{\mathrm{m}}\right)$, positif. Lors de l'émersion, le démouillage nécessite un travail de démouillage positif. On a l'habitude de représenter les grandeurs relatives au mouillage et au démouillage successifs par rapport à la même grandeur portée en ordonnée, soit $\mathrm{W}_{\mathrm{m}}$, soit $\mathrm{W}_{\mathrm{dem}}$. Dans le cas de mouillage spontané dont il s'agit ici, on utilise généralement $W_{\mathrm{dcm}}$ parce qu'il est positif. Il suffit alors de raisonner sur le travail de mouillage changé de signe. Un schéma du tracé de la force en fonction du déplacement de la lame est représenté sur la figure 2 (courbe 1) lors d'une immersion (portion abc) suivie d'une émersion (cde). Les portions ab et cd correspondent respectivement à la formation d'un ménisque quand la lame touche l'eau et à sa modification quand on inverse le sens du déplacement. Les portions bc et de sont des droites correspondant à la mesure du travail de démouillage à l'immersion puis à l'émersion (lequel est constant après la formation du ménisque), augmentée de celle du poids apparent de la lame. La parallèle à ces droites tracée à partir de l'origine $\left(\mathrm{A} \mathrm{A}^{\prime}\right)$ mesure la variation du poids apparent. La distance entre cette droite et la courbe d'immersion est égale à $\mathrm{W}_{\mathrm{dem}}$. Les deux tracés à l'émersion et à l'immersion ne coïncident pas à cause du phénomène bien connu d'hystérésis de mouillage (BLAKE et HAYNES, 1973 ; Good, 1979).

La courbe 2 de la même figure est une courbe réelle d'immersion suivie d'une émersion dans l'eau distillée d'une lame encrassée par le lactosérum à $72{ }^{\circ} \mathrm{C}$ pendant 10 minutes. Son examen appelle les remarques suivantes :

- le tracé inférieur qui correspond à l'immersion montre, au début, des irrégularités liées à l'inhomogénéité du dépôt dans cette portion, déjà mentionnée. Il suffit d'extrapoler à 0 pour obtenir le travail de démouillage $\mathbf{W}_{\text {dem }}$;

- le tracé supérieur correspondant à l'émersion, très régulier, révèle une mouillabilité à l'eau presque parfaite, qu'il s'agisse de lames nues ou encrassées par l'un quelconque des fluides laitiers étudiés. Ceci signifie que, compte tenu de la vitesse de déplacement de la lame, de l'eau reste toujours adhérente au solide après une immersion. Le tracé à l'émersion ne peut donc fournir d'informations utiles sur la nature du solide. Seuls les tracés à l'immersion seront considérés dans la suite ;

- il peut arriver que la courbe réelle, dans la portion où elle est une droite, ne varie pas comme le poids apparent. Cela signifie que $\mathrm{W}_{\text {dem }}$ varie au cours du déplacement c'est-à-dire que le dépôt est hétérogène en épaisseur, en nature, en densité superficielle.

Les mesures obtenues sur les lames encrassées dans la maquette (dépôt sur une portion seulement d'une face de la lame) font intervenir des ménisques à la limite dépôt-surface nue qui nécessiteraient une correction que les expériences nont pas permis d'évaluer avec une précision suffisante. Les mesures de mouillabilité et les spectres infrarouge sont effectués systématiquement sur les dépôts obtenus en cellule. 


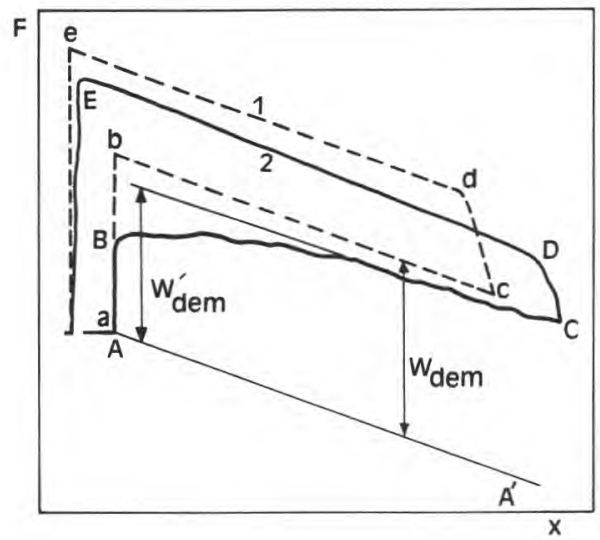

Fig. 2

Exemples de cycles de mouillage par l'eau

Examples for wetting cycles with water

1)...... - tracé schématique de la variation de force $(F)$ lors d'une immersion suivie d'une émersion ( $x$, déplacement).

1)_..... - schematic drawing of force $(F)$ variation during an immersion followed by an emersion ( $x$, displacement).

2)__ tracé expérimental pour une lame d'acier inoxydable 304 L encrassée par le lactosérum à $72{ }^{\circ} \mathrm{C}, \Delta T=0^{\circ} \mathrm{C}$ (vitesse de déplacement de la lame par rapport à l'eau : $\left.0,5 \mathrm{~cm} \cdot \mathrm{mn}^{-1}\right)$.

2) _ experimental drawing for a 304 L stainless steel slate (moving rate $=0.5$ $\mathrm{cm} \cdot \mathrm{min}^{-1}$ ) fouled with whey at $72{ }^{\circ} \mathrm{C}, \Delta T=0{ }^{\circ} \mathrm{C}$.

$W_{\text {dem }}$ et $W_{\text {dem }}^{\prime}$ : respectivement travail de démouillage à l'émersion et à l'immersion (égal au travail de mouillage mesuré à l'immersion changé de signe).

$W_{\text {dem }}$ and $W_{\text {dem }}$ : respectively work of dewetting during emersion and immersion (equal to the opposite of the work of wetting, measured during immersion).

\section{Résultats expérimentaux}

Les dépôts obtenus dans la maquette et dans la cellule sont différents quantitativement : masse de dépôt 5 fois plus faible pour le fluide "Phosphate et Calcium ", 5 fois plus forte pour le lactosérum dans le cas de l'encrassement en cellule. Le temps de séjour moyen du fluide encrassant dans le préchauffeur (deux fois plus faible dans ce cas) et des conditions hydrodynamiques différentes peuvent expliquer ces variations. Cependant, les comparaisons (entre matériaux, entre fluides, ...) sont parfaitement légitimes car les masses se classent dans le même ordre, que les dépôts soient obtenus en cellule ou en maquette. Par ailleurs, les spectres IR montrent que les dépôts obtenus sur acier inoxydable poli mécaniquement dans la maquette (DAUFIN et al., 1985) d'une part et dans la cellule d'autre part, sont très comparables qualitativement si les durées sont supérieures à $5 \mathrm{mn}$. 
La concordance entre les valeurs de $\mathrm{W}_{\mathrm{AE}}$ (Energie d'adhésion solide-eau) obtenues par mesure d'angle de contact, $(\theta)$, sur lames encrassées dans la maquette et de $W_{\text {dem }}$ sur lames encrassées dans la cellule n'est pas bonne (tabl. 1). Les valeurs de $\mathrm{W}_{\mathrm{AE}}$ déterminées à partir du $\mathrm{W}_{\mathrm{dem}}$ varient très sensiblement lorsque l'on passe du «phosphate et calcium» ou de l'ultra filtrat $\left(145 \mathrm{~mJ} \cdot \mathrm{m}^{-2}\right)$ au lait $\left(83 \mathrm{~mJ} \cdot \mathrm{m}^{-2}\right)$. Lorsque $\mathrm{W}_{\mathrm{AE}}$ est calculé à partir de la mesure de $\theta$, les variations vont dans le même sens mais elles sont beaucoup plus faibles (145 à $130 \mathrm{~mJ} \cdot \mathrm{m}^{-2}$ ). Il est vraisemblable que la mouillabilité, même partielle, des dépôts rend ceux-ci difficiles à démouiller par la bulle d'air, malgré les précautions prises lors de la mesure de $\theta$ par la méthode ANDRADE (1979). Il n'est pas étonnant que l'on mesure des valeurs voisines de l'énergie de cohésion de l'eau même quand les dépôts sont peu mouillables (lait et lactosérum à calcium complexé). Toutes ces constatations nous ont donc conduits à évaluer les caractéristiques de mouillabilité et de spectroscopie sur des dépôts obtenus après $10 \mathrm{mn}$ d'encrassement en cellule (films suffisamment minces pour la mouillabilité, suffisamment importants pour l'IR) et les masses après $20 \mathrm{mn}$ en maquette afin d'être dans le début de la phase de croissance de l'encrassement. La confrontation des données ainsi recueillies est la tradution des deux objectifs que nous nous sommes fixés.

\section{A. Encrassement par les fluides laitiers et leurs modèles}

\section{Solution de "phosphate et calcium»}

Le dépôt obtenu après l'encrassement standard est parfaitement mouillable par l'eau, et l'énergie d'adhésion dépôt/eau est égale à $146 \mathrm{~mJ} \cdot \mathrm{m}^{-2}$ (tabl. 1).

Le spectre IR révèle que c'est bien le même type de mélange d'hydrogénophosphates de calcium $\mathrm{Ca}_{4} \mathrm{H}\left(\mathrm{PO}_{4}\right)_{3}, \mathrm{nH}_{2} \mathrm{O}(\mathrm{n}=2$ et 3 ) qu'un travail précédent a déjà mis en évidence (DAUFIN et al., 1985). Le profil extrêmement détaillé (fig. 3,A) du mode $\nu_{3}\left(\mathrm{PO}_{4}\right)$ au voisinage de $1000-1100 \mathrm{~cm}^{-1}$ est caractéristique. L'adhérence au support, appréciée à partir de la facilité d'érosion lors du micropastillage, est quasi nulle, ce qui est cohérent avec les résultats de mouillabilité.

\section{Solution d'ultrafiltrat synthétique}

Les résultats de mouillabilité, identiques aux précédents, sont confirmés par la spectrométrie IR. Le composé largement majoritaire est bien un phosphate de calcium différent des hydrogénophosphates précédents. Il s'agit en fait d'une phase amorphe où le mode $\nu_{3}\left(\mathrm{PO}_{4}\right)$ fournit une bande large (fig. $3, \mathrm{~B})$ avec un seul maximum à $1070 \mathrm{~cm}^{-1}$. Le mode $v_{4}\left(\mathrm{PO}_{4}\right)$ autour de $600 \mathrm{~cm}^{-1}$ est également peu détaillé. Malgré tout, la petite bande de $875 \mathrm{~cm}^{-1}$ qui caractérise les groupements $\mathrm{HPO}_{4}^{-2}$ commence à avoir une certaine importance, le rapport des absorbances à 875 et $1040 \mathrm{~cm}^{-1}$ étant de 0,05 ; ceci est l'indice d'une tendance à la cristallisation en apatite (Heughebaert et MonTel, 1970). Effectivement, c'est ce dernier type de phase que l'on obtient systématiquement avec la maquette (fig. 3, C) ; elle n'est d'ailleurs encore qu'imparfaitement cristallisée. 


\section{TABLEAU 1}

Mouillabilité (énergie d'adhésion solideleau: $W_{A E}$ ) tirée des mesures d'angle de contact, $\theta$ (encrassement maquette) et du travail de démouillage, $W_{\text {dem }}$ (encrassement cellule), composition modélisée (\% nombre de liaisons) calculée à partir des spectres infrarouge des dépôts (cellule), masse d'encrassement pour différents fluides $\left[72{ }^{\circ} \mathrm{C}, \Delta T=0{ }^{\circ} \mathrm{C}\right.$, (maquette $20 \mathrm{mn}$; cellule $10 \mathrm{mn}$ )]

Wettability (solid-water adhesion energy: $W_{A E}$ ) from contact angle measurements, $\theta$ (fouling in scaling model) and work of dewetting,

$W_{\text {dem }}$ (fouling in cell), modelized composition (\% number of bounds) calculated from infrared spectra, masses of deposits for various fluids $\left(72{ }^{\circ} \mathrm{C}, \Delta T=0{ }^{\circ} \mathrm{C}\right)$ (scaling model : $20 \mathrm{~min}$; cell : $10 \mathrm{~min}$ )

\begin{tabular}{|c|c|c|c|c|c|c|c|c|}
\hline \multirow{2}{*}{ Fluide encrassant } & \multirow{2}{*}{\multicolumn{2}{|c|}{$\begin{array}{c}\mathrm{W}_{\mathrm{AE}}\left(\mathrm{mJ} \cdot \mathrm{m}^{-2}\right) \\
\text { calculé à partir de } \\
\theta \text { (maquette) } \quad \mathrm{W}_{\mathrm{dem}}\end{array}$}} & \multirow{2}{*}{$\begin{array}{c}\theta \\
\text { à partir de } \\
\mathrm{W}_{\mathrm{AE}} \text { moyen }\end{array}$} & \multicolumn{3}{|c|}{ Composition modélisée (\%) } & \multicolumn{2}{|c|}{ Masse $\left(\mu \mathrm{g} \cdot \mathrm{cm}^{-2}\right)$} \\
\hline & & & & Protéines & Phosphates & Lipides & Maquette & Cellule \\
\hline «Phosphate et calcium» & $145(0) 6$ & $146(1) 3$ & 0 & * & $100(0) 3$ & - & $156(7) 3$ & $40(-) 1$ \\
\hline Ultrafiltrat synthétique & $145(0) 6$ & $145(1) 4$ & 0 & $*$ & majoritaires & - & $14(5) 3$ & $18(-) 1$ \\
\hline Lactosérum & $144(1) 6$ & $112(6) 10$ & 56 & $78(3) 4$ & $21(3) 4$ & $1(2) 4$ & $13(4) 3$ & $63(3) 3$ \\
\hline $\begin{array}{l}\text { Lactosérum } \\
\text { (calcium complexé) }\end{array}$ & $136(3) 6$ & $98(4) 3$ & 71 & $93(0,4) 2$ & $5(0,8) 2$ & $2(0,4) 2$ & $8(2) 3$ & - \\
\hline Lait & $130(3) 6$ & $83(1) 3$ & 83 & $95(-) 1$ & $2(-) 1$ & $3(-) 1$ & $5(3) 3$ & - \\
\hline
\end{tabular}

Les résultats sont indiqués de la façon suivante : valeur moyenne (écart-type) nombre de mesures.

* La composition modèle n'a pas de sens avec ces solutions qui ne contiennent pas de protéines. 


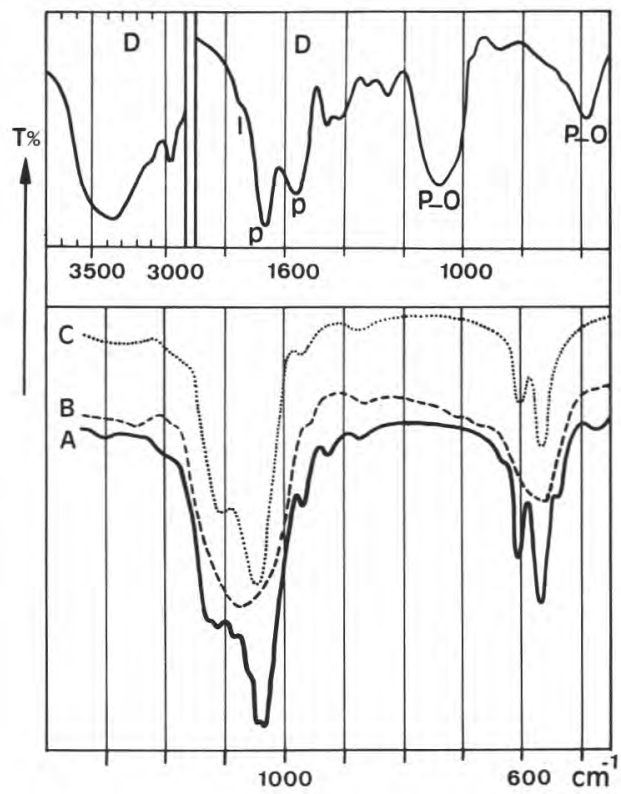

Fig. 3

Extraits de spectres infrarouges ( $T$, Transmission \%; nombre d'ondes en $\left.\mathrm{cm}^{-1}\right)$ de dépôts recueillis après encrassement $\left(72{ }^{\circ} \mathrm{C} ; \Delta T=0{ }^{\circ} \mathrm{C}\right)$ par différents fluides pendant 10 minutes.

Extracts from I.R. Spectra (T, Transmission \%; wave number $\mathrm{cm}^{-1}$ ) after $10 \mathrm{~min}$ fouling with some fluids $\left(72{ }^{\circ} \mathrm{C}, \Delta T=0^{\circ} \mathrm{C}\right)$.

A phosphate + calcium

$B$ ultrafiltrat (cellule)/artificial ultrafiltrate (cell)

$C$ ultrafiltrat (maquette)/artificial ultrafiltrate (scaling model)

$D$ lactosérum doux/sweet whey

$l=$ lipides/lipids ; $p=$ protéines/proteins

$P-O=$ phosphates $/$ phosphates.

3. Lactosérum doux de pâte cuite

La composition des dépôts modélisée à partir des spectres infrarouge donne : protéine $78 \%$, phosphate $21 \%$, lipide $1 \%$ (tabl. 1), proportions voisines de celles obtenues avec la maquette. Le plus remarquable au niveau structural est le caractère décidément amorphe du phosphate de calcium (fig. 3 , D). Le rapport des absorbances à 875 et $1040 \mathrm{~cm}^{-1}$ est plus petit $(\simeq 0,03)$. Le profil du spectre montre également la faible contribution des lipides par rapport aux protéines.

Pour ces films beaucoup plus complexes que les précédents, l'énergie d'adhésion devient égale à $112 \mathrm{~mJ} \cdot \mathrm{m}^{-2}$. 
Il est intéressant de noter que, dans le cas du lactosérum, la tension superficielle de l'eau dans laquelle a lieu le cycle de mouillage (égale au départ à environ $72 \mathrm{mN} \cdot \mathrm{m}^{-1}$ ) est systématiquement abaissée à environ $65 \mathrm{mN} \cdot \mathrm{m}^{-1}$ après le cycle. Il semblerait que le dépôt de lactosérum contamine l'eau, ce qui pourrait être dû à une solubilisation de protéines tensioactives par exemple. Le mécanisme corespondant n'est cependant pas évident puisqu'on n'observe pas le même effet avec le lait ou le lactosérum complexé.

\section{Lactosérum à calcium complexé}

Les dépôts observés sont irréguliers et très sensiblement moins abondants que dans le cas précédent. La courbe de mouillabilité, très irrégulière elle aussi, dénote une diminution de $\mathrm{W}_{\mathrm{AE}}\left(98 \mathrm{~mJ} \cdot \mathrm{m}^{-2}\right)$ par rapport au lactosérum. La présence de très faibles quantités de phosphates $(5 \%)$, malgré la complexation du calcium, est un phénomène intéressant car il confirmerait bien une interaction complexante entre les phosphates et les protéines lesquelles sont en outre largement majoritaires (93\%) (tabl. 1). Un excès important de complexant fait effectivement disparaître les phosphates totalement.

\section{Lait écrémé reconstitué à partir de poudre "Low Heat"}

Aucune mesure n'est nécessaire pour constater que le dépôt est beaucoup plus faible qu'avec le lactosérum. Le tracé de mouillage fait apparaître un $\mathrm{W}_{\mathrm{AE}}$ nettement plus petit $\left(83 \mathrm{~mJ} . \mathrm{m}^{-2}\right)$ qu'avec le lactosérum. Les phosphates sont en beaucoup plus faible proportion ( $2 \%$ ) tandis que les protéines sont fortement majoritaires (95\%) et les lipides un peu plus importants (3\%) (tabl. 1).

\section{B. Influence du procédé de nettoyage}

\section{Etude de lames non encrassées après différents nettoyages}

Les énergies de démouillage dépendent du temps qui s'écoule entre le dernier rinçage après nettoyage et le tracé du cycle. Les mesures sont donc faites immédiatement après rinçage, la lame étant "mouillée " comme dans le processus industriel d'encrassement.

Une lame encrassée par le lactosérum dans les conditions standard puis nettoyée «laiterie», n'est pas parfaitement mouillable $\left(\theta=70^{\circ}\right)$ et $\mathrm{W}_{\mathrm{AE}}$, égal à $102 \mathrm{~mJ} \cdot \mathrm{m}^{-2}$, est nettement inférieur à l'énergie de cohésion de l'eau $\left(146 \mathrm{~mJ} \cdot \mathrm{m}^{-2}\right)$, mesurée dans le cas d'une lame analogue nettoyée au mélange sulfochromique. Cette lame, nettoyée ensuite par immersion dans le trichloréthylène, présente une diminution de masse de $32 \mu \mathrm{g}$. Un traitement analogue par l'hexane puis l'éthanol, effectué ensuite, fait apparaître une nouvelle diminution de $33 \mu \mathrm{g}$. Ceci signifierait donc que la lame nettoyée «laiterie » est encore souillée de lipides ou lipoprotéines, solubles dans les solvants utilisés. On a donc tenté d'analyser par spectrométrie IR cet éventuel dépôt résiduel. Le spectre enregistré après micropastillage du dépôt qui subsiste, révèle, par rapport à un dépôt de lactosérum obtenu sur une lame ayant subi le nettoyage au mélange sulfochromique, un net enrichissement en lipides (13\% au lieu de $1 \%$ ) tandis que la proportion de protéines est pratiquement inchangée. Les phosphates, paradoxalement toujours visibles, ont beaucoup diminué (11\% au 
TABLEAU 2

Masses de dépôts pour différents matériaux encrassés par le lactosérum $\left(72{ }^{\circ} \mathrm{C}\right.$. $\Delta T=0^{\circ} \mathrm{C}, 20 \mathrm{mn}$ ) et caractéristiques de mouillage (angle de contact, $\theta$ et énergie d'adhésion, $W_{A E}$ ) avant encrassement

Masses of deposits for materials fouled by whey $\left(72{ }^{\circ} \mathrm{C}, \Delta T=O^{\circ} \mathrm{C}, 20 \mathrm{~min}\right)$ and before fouling characteristics of wetting (contact angle, $\theta$ and adhesion energy, $W_{A E}$ )

\begin{tabular}{|c|c|c|c|c|}
\hline Matériau & $\begin{array}{c}\text { Etat de surface } \\
\text { (procédé de nettoyage) }\end{array}$ & $\theta\left({ }^{\circ}\right)$ & $\begin{array}{c}\mathrm{W}_{\mathrm{AE}_{\mathrm{nu}}} \\
\left(\mathrm{mJ} \cdot \mathrm{m}^{-2}\right)\end{array}$ & $\begin{array}{l}\text { Masse et } \\
\text { écart type } \\
\left(\mu \mathrm{g} . \mathrm{cm}^{-2}\right)\end{array}$ \\
\hline $\begin{array}{l}\text { Acier } \\
\text { inoxydable } \\
304 \mathrm{~L}\end{array}$ & $\begin{array}{ll}\text { poli } & \begin{array}{l}\text { (alcool) } \\
\text { (sulfochromique) } \\
\text { BLG }\end{array} \\
& \begin{array}{l}\text { (alcool) } \\
\text { (sulfochromique) }\end{array} \\
\text { électrolytique (alcool) }\end{array}$ & $\begin{array}{r}0 \\
14\end{array}$ & $\begin{array}{l}146(1) 8 \\
144(4) 4\end{array}$ & $\begin{array}{l}56(4) 3 \\
50(5) 3 \\
45(20) 3 \\
66(8) 3\end{array}$ \\
\hline $\begin{array}{l}\text { Téflon } \\
\text { massif }\end{array}$ & $\begin{array}{ll}\text { brut } & \begin{array}{l}\text { (standard) } \\
\text { (sulfochromique) } \\
\text { poli }\end{array} \\
\text { (sulfochromique) }\end{array}$ & $\begin{array}{r}20 \\
0\end{array}$ & $\begin{array}{l}142(3) 5 \\
146(1) 3\end{array}$ & $\begin{array}{l}55(13) 3 \\
55(3) 3 \\
-\end{array}$ \\
\hline Verre & $\begin{array}{l}\text { (alcool) } \\
\text { (sulfochromique) }\end{array}$ & 12 & $144(1) 4$ & $\begin{array}{l}42(2) 3 \\
35(1) 3\end{array}$ \\
\hline $\begin{array}{l}\text { Résine } \\
\text { - Silicone } \\
\text { - Fluorée }\end{array}$ & $\begin{array}{l}\text { (alcool) } \\
\text { (alcool) }\end{array}$ & & & $\begin{array}{l}55(35) 3 \\
66(50) 3\end{array}$ \\
\hline Polyéthylène & (alcool) & & & $36(12) 3$ \\
\hline $\begin{array}{l}\text { ASG oxydé } \\
\text { anodiquement }\end{array}$ & (alcool) & & & $36(15) 3$ \\
\hline
\end{tabular}

Les résultats sont donnés de la façon suivante : valeur moyenne (écart-type) nombre de mesures.

lieu de $23 \%$ ). Le nettoyage « laiterie » apparaît donc insuffisant pour éliminer toute contamination provoquée par un dépôt précédent. La question se pose alors de savoir si cette souillure influe sur l'encrassement ultérieur par le lactosérum.

\section{Encrassement par le lactosérum de lames diversement nettoyées}

Des lames d'acier inox $304 \mathrm{~L}$, préalablement encrassées par du lactosérum dans des conditions standard subissent divers nettoyages (mélange sulfochromique, «laiterie », ou « hexane - éthanol » suivi d'un traitement « laiterie » puis d'un lavage " hexane - éthanol "). On les encrasse à nouveau par du lactosérum dans les conditions standard et on mesure le travail de démouillage pour chacune d'elles. Les résultats font apparaître des polarités différentes, traduites par des valeurs de $W_{\mathrm{AE}}$ respectivement égales à 111,89 et $123 \mathrm{~mJ} \cdot \mathrm{m}^{-2}$. On constate également que le dépôt sur une lame nettoyée au mélange sulfochromique a une masse moyenne de $870 \mu \mathrm{g}$ (soit $60 \mu \mathrm{g} . \mathrm{cm}^{-2}$ ) alors qu'on obtient 


\section{TABLEAU 3}

Encrassement de quelques matériaux par le lactosérum $\left(72{ }^{\circ} \mathrm{C}, \Delta T=0^{\circ} \mathrm{C}\right):$ masses $(20 \mathrm{mn}$ en maquette $)$, composition modélisée calculée à partir des spectres infrarouges et énergie d'adhésion, $W_{A E}(10 \mathrm{mn}$ en cellule)

Fouling of some materials with whey $\left(72{ }^{\circ} \mathrm{C}, \Delta T=0^{\circ} \mathrm{C}\right.$ ) : masses (after 20 min in scaling model), modelized composition calculated from infrared spectra and solid-water adhesion energy, $W_{A E}(10 \mathrm{~min}$ in cell $)$

\begin{tabular}{l|c|c|c|c|c|c}
\hline \multirow{2}{*}{\multicolumn{1}{c|}{ Matériau }} & \multicolumn{2}{|c|}{$\mathrm{W}_{\mathrm{AE}}\left(\mathrm{mJ} . \mathrm{m}^{-2}\right)$} & \multicolumn{3}{c}{$\begin{array}{c}\text { Composition modélisée } \\
(\% \text { nombre de liaisons) }\end{array}$} & $\begin{array}{c}\text { Masse } \\
(\mu \mathrm{g} . \mathrm{cm}\end{array}$ \\
\cline { 2 - 8 } & $\mathrm{nu}$ & encrassé & Protéines & Phosphates & Lipides \\
\hline Acier inoxydable poli sulfochromique & $146(1) 8$ & $112(6) 10$ & $78(3) 4$ & $21(3) 4$ & $1(2) 4$ & $50(5) 3$ \\
\hline Téflon brut sulfochromique & $142(3) 5$ & $115(1) 4$ & $79(-) 1$ & $17(-) 1$ & $4(-) 1$ & $55(13) 3$ \\
\hline Verre sulfochromique & $144(1) 4$ & $83(2) 2$ & $77(-) 1$ & $22(-) 1$ & $1(-) 1$ & $35(1) 3$ \\
\hline
\end{tabular}

Les résultats sont donnés de la façon suivante : valeur moyenne (écart-type) nombre de mesures. 
$910 \mu \mathrm{g}$ soit $4 \%$ de plus avec un nettoyage «laiterie ». C'est donc en général sur un dépôt résiduel faible mais bien visible que se font les encrassements industriels.

\section{Influence de l'état de surface de l'acier inoxydable}

Les propriétés des lames d'acier $304 \mathrm{~L}$, poli ou brut de laminage glacé (BLG), nettoyées au mélange sulfochromique, sans dépôt, ne sont que légèrement différentes. En effet, $\mathrm{W}_{\mathrm{AE}}$ est égal à $144 \mathrm{~mJ} \cdot \mathrm{m}^{-2}$ pour une lame BLG, 146 pour une lame polie (tabl. 2).

Pour les lames polies, le lactosérum utilisé dans les conditions standard donne des encrassements reproductibles dont l'énergie d'adhésion moyenne est $112 \mathrm{~mJ} \cdot \mathrm{m}^{-2}$ (écart type $=3 \mathrm{~mJ} \cdot \mathrm{m}^{-2}$ ) tandis que les masses sont assez reproductibles (masse moyenne $=56 \mu \mathrm{g} \cdot \mathrm{cm}^{-2}$; écart type $=4 \mu \mathrm{g} \cdot \mathrm{cm}^{-2}$ ). Pour les lames BLG, les tracés sont peu reproductibles. En effet, au cours de 5 répétitions, $\mathrm{W}_{\mathrm{AE}}$ varie de 93 à $115 \mathrm{~mJ} \cdot \mathrm{m}^{-2}$ tandis que les masses sont très dispersées (écart type $=20 \mu \mathrm{g} \cdot \mathrm{cm}^{-2}$ ) autour d'une masse moyenne $45 \mu \mathrm{g} \cdot \mathrm{cm}^{-2}$ (tabl. 2).

\section{Influence du matériau}

Dans le but de préciser le rôle éventuel de l'eau présente avant l'encrassement sur la paroi mouillée, nous avons examiné quelques matériaux dont les mouillabilités «à $\sec »$ sont très différentes, tels l'acier poli, le Téflon et le verre. Pour chacun d'eux, nous avons étudié les propriétés, d'une part de la lame nue (nettoyée au mélange sulfochromique, mouillée) et d'autre part de la même lame encrassée par le lactosérum dans les conditions standard.

\section{Acier inoxydable $304 \mathrm{~L}$}

Après nettoyage au mélange sulfochromique, la mouillabilité de la lame polie mesurée immédiatement après rinçage est presque parfaite. L'encrassement par le lactosérum donne un dépôt moins polaire puisque $\mathrm{W}_{\mathrm{AE}}$ est égal à $112 \mathrm{~mJ} . \mathrm{m}^{-2}$ (tabl. 3).

La composition du dépôt est $78 \%$ en protéines, $21 \%$ pour les phosphates et $1 \%$ de lipides. La masse d'encrassement est de $50 \mu \mathrm{g} . \mathrm{cm}^{-2}$ (tabl. 3).

\section{Téflon}

Le Téflon massif est étudié à l'état brut et à l'état poli 800 . Au début de la mesure, les valeurs de $\mathrm{W}_{\mathrm{AE}}$ sont élevées [142 (brut) et 146 (poli) $\mathrm{mJ} . \mathrm{m}^{-2}$ ] puis diminuent en raison d'un "séchage" de la lame en cours de mesure. Puisque l'encrassement se fait sur une lame complètement mouillée, nous retiendrons les valeurs à l'origine, soit 142 à $146 \mathrm{~mJ} \cdot \mathrm{m}^{-2}$ avant et après polissage respectivement. Les encrassements par le lactosérum sont voisins. Ils présentent une énergie d'adhésion $\mathrm{W}_{\mathrm{AE}}$ égale en moyenne à $115 \mathrm{~mJ} . \mathrm{m}^{-2}$, pratiquement égale à celle qu'on observe avec l'acier poli $\left(112 \mathrm{~mJ} \cdot \mathrm{m}^{-2}\right)$. La composition correspond à : protéines $79 \%$, phosphates $17 \%$, lipides $4 \%$. L'encrassement moyen atteint $55 \mu \mathrm{g} \cdot \mathrm{cm}^{-2}$ (tabl. 2 et 3 ). 


\section{Verre}

La lame de verre nettoyée au mélange sulfochromique est parfaitement mouillable : $\mathrm{W}_{\mathrm{AE}}=144 \mathrm{~mJ} \cdot \mathrm{m}^{-2}$. La même lame encrassée par le lactosérum présente un tracé en dents de scie, typique d'un dépôt apolaire. $\mathrm{W}_{\mathrm{AE}}$ est égal en moyenne à $83 \mathrm{~mJ} \cdot \mathrm{m}^{-2}$, valeur la plus basse que nous ayons mesurée avec le lactosérum. La composition du dépôt est très voisine de celle qui a été obtenue pour l'acier inoxydable: protéines $77 \%$, phosphates $22 \%$, lipides $1 \%$, bien que la masse moyenne de l'encrassement soit plus faible $\left(35 \mu \mathrm{g} . \mathrm{cm}^{-2}\right.$ ) (tabl. 2 et 3 ).

\section{Autres matériaux}

Sur les deux types de films minces (résine silicone et résine fluorée) les masses sont très dispersées (écart-type 35 et $50 \mu \mathrm{g} . \mathrm{cm}^{-2}$ respectivement), rendant la comparaison avec la surface témoin très difficile en dépit de valeurs moyennes de masses (respectivement 55 et $66 \mu \mathrm{g} . \mathrm{cm}^{-2}$ pour la résine silicone et la résine fluorée) voisines de celle de la surface témoin polie (tabl. 2). Pour les deux autres matériaux utilisés, polyéthylène $\left(36 \mu \mathrm{g} . \mathrm{cm}^{-2}\right)$ et alliage d'aluminium anodisé $\left(36 \mu \mathrm{g} \cdot \mathrm{cm}^{-2}\right)$, les masses sont voisines de celles trouvées pour le verre.

\section{Discussion}

\section{A. Caractéristiques des films d'encrassement: apport des mesures de $W_{A E}$ à la compréhension du mécanisme}

Le dépôt obtenu avec la solution de «phosphate et calcium » est très polaire, et son énergie d'adhésion $\left(146 \mathrm{~mJ} \cdot \mathrm{m}^{-2}\right)$ correspond à l'énergie de cohésion de l'eau. Donc, la couche est pleine d'eau ou l'interaction dépôt/eau est égale à l'énergie de cohésion de l'eau. Un tracé enregistré avec l'un des dépôts, $48 \mathrm{~h}$ après la première mesure, quand l'eau présente s'est évaporée, montre une diminution de la mouillabilité. Cependant, $\mathrm{W}_{\mathrm{AE}}$ reste élevé (118 $\mathrm{mJ} \cdot \mathrm{m}^{-2}$ ), résultat qu'on pouvait attendre en présence d'un dépôt purement inorganique identifié par IR à un mélange d'hydrogénophosphates de calcium. Les phosphates de calcium complexes (apatite en voie de cristallisation) obtenus avec l'ultrafiltrat conduisent à une mouillabilité de la surface voisine de celle observée précédemment.

Le film d'encrassement par le lactosérum conduit à une diminution des interactions avec l'eau $\left(\mathrm{W}_{\mathrm{AE}}=112 \mathrm{~mJ} \cdot \mathrm{m}^{-2}\right)$. Les films minces de protéines, lipides et phosphates, dont on peut raisonnablement fixer l'épaisseur à un ordre de grandeur d'une centaine d'angstroems (expériences en spectrophotométrie de photoélectrons $\mathrm{X}$ ), forment donc une couche partiellement hydrophobe. Ceci s'explique si les groupes polaires des protéines se fixent préférentiellement sur le support soit directement soit par complexation avec les espèces ioniques «phosphate et calcium », les sites hydrophobes se trouvant rejetés à l'extérieur. 
Dans le cas du lactosérum à calcium complexé, la diminution de $\mathrm{W}_{\mathrm{AE}}$ par rapport au lactosérum $\left(98 \mathrm{~mJ} \cdot \mathrm{m}^{-2}\right.$ au lieu de $\left.112 \mathrm{~mJ} \cdot \mathrm{m}^{-2}\right)$ signifie une polarité plus faible du dépôt. Les protéines se fixeraient toujours par les groupes polaires mais il y a une diminution des composés inorganiques en raison de la complexation du calcium par le citrate et le caractère hydrophobe augmente.

Le dépôt obtenu avec le lait, où les protéines sont maintenant nettement majoritaires (tabl. 1), est plus apolaire que dans le cas du lactosérum. Ceci pourrait se justifier par la présence d'une quantité relative plus importante de protéines et de lipides (tabl. 1) susceptibles d'entraîner un caractère plus hydrophobe. L'effet inhibiteur des caséines sur la précipitation du phosphate de calcium ainsi que l'absence de variations dans la composition et la faible croissance des masses au cours du temps (DAufin et al., 1985) expliquent cette composition des dépôts.

\section{B. Influence du nettoyage}

Le nettoyage «laiterie» ne suffit pas à éliminer toute contamination de la surface provoquée par un dépôt précédent, ce qui, logiquement, modifie l'encrassement ultérieur. D'une manière plus générale, la polarité du dépôt et donc sa structure varient suivant le procédé de nettoyage. La polarité et le caractère hydrophile décroissent quand on passe du nettoyage par solvants et produits acido-basiques suivis de solvants, au mélange sulfochromique puis au nettoyage «laiterie ».

L'effet observé pour les contaminations restant après nettoyage «laiterie » se traduit par de faibles modifications de l'encrassement mesurées après une durée aussi courte que 10 minutes. Une étude systématique pour des durées plus longues mériterait d'être entreprise.

Quelques essais de prédépôt de lactosérum ou d'ultrafiltrat sur acier avant encrassement sont effectués pour examiner le rôle de ces dépôts sur un encrassement ultérieur. Un prédépôt de lactosérum est réalisé dans les conditions standard pendant 20 minutes, puis la lame est rincée à l'eau. La masse du dépôt est alors $57 \mu \mathrm{g} . \mathrm{cm}^{-2}$. Puis la lame subit un second encrassement de 20 minutes, toujours dans les conditions standard. La masse supplémentaire déposée est $130 \mu \mathrm{g} . \mathrm{cm}^{-2}$, c'est-à-dire beaucoup plus importante que dans les 20 premières minutes. Ces résultats sont à rapprocher de ceux publiés par RoignANT et al., (1983). Ces auteurs ont mesuré les masses d'encrassement par le lactosérum du même acier, dans des conditions expérimentales un peu différentes pendant des durées allant jusqu'à 90 minutes. Ils observent une augmentation importante de la vitesse d'encrassement avec le temps, c'est-àdire avec la masse déjà déposée. Une étude de l'encrassement par du lait pendant plusieurs heures avait permis à Lalande et CORRIEU (1981) de proposer une cinétique autocatalytique d'ordre 0,5 . Le dépôt formé au cours d'une expérience préalable de 20 minutes jouerait donc le même rôle que la masse déjà déposée au cours des 20 premières minutes de la même expérience. Cet effet pourrait résulter d'une augmentation de "l'aire réactive " au fur et à mesure de la formation du dépôt, très supérieure à celle de la surface en acier inoxydable. On observe le même phénomène, amplifié, dans le cas d'un prédépôt d'ultrafiltrat, ce qui pourrait s'expliquer par la présence d'apatites en voie de cristallisation, composés favorables à un processus de croissance. 
Ces résultats sont en contradiction avec ceux de Lund et BrxBy (1975) qui constatent une diminution de l'encrassement par du lait lorsque la surface de leur échangeur tubulaire est recouverte d'un film de phosphate tricalcique. Ce dépôt initial divise par deux le coefficient de transfert de chaleur mais la valeur de ce dernier reste ensuite constante pendant $3 \mathrm{~h}$, ce qui n'est pas le cas lorsque la surface initiale est nue. On peut se demander si ce résultat ne rend pas compte d'une diminution de la température de paroi sous l'influence du prédépôt, cette possibilité ayant été exclue de notre expérimentation par l'utilisation d'un gradient de température nul.

\section{Influence de l'état de surface}

Qu'elles soient polies ou non, les lames d'acier sont très mouillables (tabl. 2) et $W_{A E}$ varie peu d'un essai à l'autre. Les énergies d'adhésion avec l'eau de ces lames encrassées sont toutes nettement plus petites. La comparaison des courbes de mouillabilité montre essentiellement une mauvaise répétabilité pour les lames BLG, au contraire de ce qui est observé pour les lames polies, en accord avec les fortes dispersions des masses : entre 20 et $70 \mu \mathrm{g} . \mathrm{cm}^{-2}$. Il faut de plus remarquer que les valeurs de $\mathrm{W}_{\mathrm{AE}}$ ou de masses d'encrassement obtenues pour la lame BLG encadrent la valeur qui correspond à la lame polie.

Les opinions exprimées par les différents chercheurs dans la bibliographie sur le rôle du degré de polissage sont très variées. Daufin et al. (1977) ont montré que l'acier inoxydable BLG ne se comporte par différemment d'une surface polie mécaniquement (poli 180: granulométrie $80 \mu \mathrm{m}$ ) plus rugueuse que celle utilisée dans ce travail. Pour MarriotT (1971), un meilleur polissage réduit l'encrassement pour tout échangeur de chaleur. GoRdon et al. (1968) préconisent un tel traitement à l'issue d'essais réalisés avec du lait à $82^{\circ} \mathrm{C}$ en recirculation. NovaK (1981) estime que le polissage augmente la durée avant apparition de l'encrassement. WennERBERG (1981) pense que l'adhérence du dépôt est moins forte sur une surface polie sans que celui-ci soit diminué. LiNG et LUND (1978), travaillant avec de l'albumine d'œuf, ne trouvent aucune différence entre diverses finitions. Enfin, nous n'avons pas retrouvé pour les surfaces polies électrolytiquement (tabl. 2), la très spectaculaire réduction d'encrassement observée pour la liqueur noire de pâte à papier par NorDiN et WESTERGREN (1981) ni la légère diminution d'encrassement que nous avions mesurée par pesage et par dénombrement bactérien (DAUFIN et al., 1977).

Ces résultats apparemment plus ou moins contradictoires pourraient s'expliquer par des phénomènes d'hystérésis de mouillage bien connus qui justifieraient des différences importantes des angles de mouillage donc des valeurs de $\mathrm{W}_{\mathrm{AE}}$, provoquées, par exemple, par de légères modifications hydrodynamiques ou structurales locales. Par ailleurs, ils pourraient signifier l'absence du rôle de l'état de surface, les différences s'estompant avec la croissance de l'encrassement.

\section{Influence de la nature du matériau}

La comparaison des résultats obtenus avec des lames d'acier poli, de Téflon massif et de verre nettoyées puis encrassées de la même façon devait 
permettre de dégager l'influence de la nature du matériau. $\mathrm{W}_{\mathrm{AE}}$ est plus petit pour une lame d'acier polie encrassée par le lactosérum $\left(112 \mathrm{~mJ} . \mathrm{m}^{-2}\right)$ que pour cette lame nue $\left(146 \mathrm{~mJ} \cdot \mathrm{m}^{-2}\right)$. Ce résultat dénote une diminution des interactions du solide avec l'eau. Comme nous l'avons déjà mentionné il signifierait une surface plus hydrophobe que celle de la lame avant encrassement, ce qui s'explique si les groupes polaires des protéines se fixent préférentiellement sur le support, ce qui est attendu puisque la surface de l'acier est elle-même très polaire.

Les courbes obtenues à partir du Téflon massif posent plus de questions. En effet, la mouillabilité de lames nues de Téflon avant encrassement, respectivement brut ou poli, révèle que la surface de ce matériau, réputé non mouillable, apparaît comme très mouillable par l'eau. Cependant, l'allure des courbes de mouillabilité est différente de celle relative à l'acier inoxydable BLG ou poli. Tandis que la mouillabilité ne varie pas au cours de l'immersion de la lame d'acier, $W_{\mathrm{AE}}$ diminue continûment avec le Téflon. Ceci signifie que le Téflon, poli ou non, est initialement recouvert d'un film d'eau et $\mathrm{W}_{\mathrm{AE}}$ est voisin de l'énergie de cohésion de l'eau, mais ce film «s'essore " ou sèche durant le temps que dure l'immersion et $\mathrm{W}_{\mathrm{AE}}$ diminue un peu. C'est donc sur un film d'eau que doit se faire le dépôt. Ceci semble bien être vérifié par le fait que $\mathrm{W}_{\mathrm{AE}}$ correspondant à une lame de Téflon (polie ou non), encrassée par le lactosérum, est égal en moyenne à $115 \mathrm{~mJ} \cdot \mathrm{m}^{-2}$, valeur très proche de celle qui a été trouvée pour l'acier. Les encrassements moyens sont eux-mêmes pratiquement identiques : $50 \mu \mathrm{g} . \mathrm{cm}^{-2}$ pour l'acier, 55 pour le Téflon. La nature des dépôts devrait donc être voisine, ce que confirment les spectres IR révélant toutefois un peu plus de lipides sur le Téflon. Ces résultats sont à rapprocher de ceux rapportés par GoRdon et al. (1968) indiquant que le Téflon retient une masse d'encrassement par le lait $\left(82^{\circ} \mathrm{C}\right)$ plus forte que celle qui est relative à l'acier inoxydable (sans doute liée à une température de paroi sur Téflon plus élevée dans leurs essais) mais qui s'éliminerait plus facilement après · séchage.

Le verre nettoyé au mélange sulfochromique est un matériau très polaire $\left(\mathrm{W}_{\mathrm{AE}}=144 \mathrm{~mJ} \cdot \mathrm{m}^{-2}\right)$. Mais il présente, par rapport à l'acier, la particularité d'avoir des sites anioniques $\mathrm{SiO}$. On est conduit à penser que les ions $\mathrm{Ca}^{2+}$ se fixent sur ces sites et se lient par ailleurs aux phosphates ainsi qu'aux protéines plus ou moins dénaturées présentes dans le film d'eau; les groupements carboxylates des protéines sont certainement impliqués dans ce type de liaison, mais d'autres fonctions peuvent également intervenir, tels les amides par exemple. Dans ce cas, la partie externe du dépôt devrait présenter un caractère hydrophobe assez marqué. C'est effectivement le résultat trouvé puisque l'énergie d'adhésion est faible $\left(83 \mathrm{~mJ} . \mathrm{m}^{-2}\right)$ et l'angle de mouillage petit $\left(\theta=81^{\circ}\right)$. Le tracé en dents de scie est, en outre, caractéristique d'un dépôt apolaire, dû à des phénomènes d'hystérésis particulièrement marqués lorsque l'angle de contact est voisin de $90^{\circ} \mathrm{C}$. Le film d'eau qui recouvre la surface solide n'empêche donc pas des interactions physiques ou chimiques locales entre les constituants du fluide laitier et la surface du matériau. Mais Ia composition du dépôt déduite du spectre IR est pratiquement identique à celle du dépót sur acier, tandis que la masse d'encrassement $\left(35 \mu \mathrm{g} . \mathrm{cm}^{-2}\right)$ est un peu plus petite (tabl. 2). Les interactions entre les composants du lait, au cours du processus d'encrassement, sont donc prédominantes. Il est donc normal que les dépôts se révèlent très voisins, à la fois quant à leur composi- 
tion et quant à la masse d'encrassement. Ce résultat est cohérent avec les expériences de LALANDE et al. (1981) qui n'ont trouvé aucune différence entre les vitesses d'encrassement, par du lait, de surfaces d'échange de chaleur en matériaux divers : alliage d'aluminium $\left(\mathrm{A}_{7} \mathrm{G}_{3}\right)$ nu ou revêtu d'une résine éthyl méthyl cétone, acier inoxydable $316 \mathrm{Ti}, 304 \mathrm{~L}$ et $434 \mathrm{Ti}$.

Le caractère amphiphile de certains constituants du lactosérum (protéines, lipoprotéines, lipides, phosphates de calcium complexés par les protéines ou les lipoprotéines) semble être un élément crucial puisque ces derniers sont susceptibles d'interagir avec tout support solide, qu'il soit hydrophile ou hydrophobe.

\section{Conclusion}

Les caractéristiques de mouillabilité des dépôts obtenus à partir de différents fluides laitiers et de leurs modèles sont très cohérentes avec les résultats qu'on peut déduire des spectres IR et de la masse des dépôts.

C'est logiquement l'étape initiale d'adsorption de la ou des toutes premières couches qui reste le processus d'amorçage de l'encrassement. En effet, en l'absence d'air, un amphiphile en solution dans l'eau peut s'adsorber sur une paroi solide si l'énergie d'interaction avec cette paroi est plus importante que l'interaction avec l'eau avoisinante. C'est cette compétition avec l'eau qui expliquerait pourquoi la présence constante d'un film aqueux recouvrant la surface du solide - qui semble découler des expériences - tend à uniformiser les étapes ultérieures de l'encrassement. Il reste que des interactions physiques ou chimiques locales entre constituants du fluide laitier et paroi ne sont pas exclues initialement.

En effet, quel que soit le mécanisme d'amorçage, l'effet s'en estompe très vite. Les interactions entre constituants du fluide et composés déjà adsorbés se révèlent prédominantes au cours du processus de croissance du dépôt. Ces dernières favoriseraient la formation, entre les protéines en cours de dénaturation, les ions calcium et les phosphates, d'un véritable complexe peu soluble dont les propriétés amphiphiles permettraient la fixation sur tout support hydrophile ou hydrophobe. C'est donc sur ce complexe que l'action pratique peut porter, notamment par complexation des ions calcium. Par contre, il ne paraît pas utile de recommander industriellement d'agir sur l'état de surface ou sur la nature du matériau puisque ces deux paramètres se révèlent sans incidence sur la réduction de l'encrassement sauf si l'on considère que les problèmes de nettoyage et de désinfection ne sont pas disjoints de ces deux paramètres, ce qui reste à démontrer. Par ailleurs, puisque l'existence d'un premier dépôt accélère inévitablement l'encrassement ultérieur, il pourrait être avantageux de rechercher l'utilisation de procédures de nettoyage laissant les surfaces très propres (très forte mouillabilité à l'eau). Ces mesures seraient inévitablement coûteuses (produits chimiques hautement actifs, ...) sans que l'on puisse affirmer aujourd'hui qu'elles sont susceptibles d'entraîner une réduction de l'encrassement tout au long de la durée d'un traitement thermique (plusieurs heures), par suite de la suppression du dépôt résiduel initial. 
Par contre, toute cause susceptible de perturber la formation du premier dépôt qui s'effectue essentiellement au travers d'un film d'eau pourrait être bénéfique dans la lutte contre l'encrassement. Une action sur l'écoulement au voisinage de la paroi provoquant une diminution du temps de séjour des constituants encrassants pourrait diminuer les interactions chimiques ou physiques responsables de la croissance des dépôts.

Reçu le $1^{\text {er }}$ août 1986.

Accepté pour publication le 29 janvier 1987.

\section{Remerciements}

Ce travail a bénéficié de l'aide financière du Ministère de l'Industrie et de la Recherche. Contrat d'Aide à la Recherche 83 C 0165. Action Technologie Alimentaire et Agricole.

Nous remercions bien vivement notre collègue $M$. RoIGNANT pour sa contribution à la mise au point des techniques de mesure de tension superficielle et d'angle de contact.

\section{Références bibliographiques}

Andrade J.D., King R.N., Gregonis D.E., Coleman D.L., 1979. Surface characterization of poly (hydroxyethylmethacrylate) and related polymers. I. Contact angle methods in water. $J$. Polym. Sci., C., 66, 313-336.

BAIER R., 1981. Modification of surfaces to reduce fouling and/or improve cleaning. In : Fundamentals and applications of surface phenomena associated with fouling and cleaning in food processing. Ed. Hallstrom B., Lund D.B., Tragardh Ch., Lund University, 168-189.

Blake T.D., Haynes J.M., 1973. Contact angle hysteresis. Prog. Surf. Membrane Sci., 6, 125-138.

Daufin G., Kerhervé L., Richard J., 1977. Influence de l'état de surface de l'acier inoxydable sur son aptitude au nettoyage et sur sa sensibilité à la corrosion par piqûres. Ind. Alim. Agric., 94 (1), 43-51.

Daufin G., Roignant M., Michel F., Kerhervé F.L., Dupeyrat M., Labbé J.P., Pagetti J., Quemerais A., Brulé G., 1985, Matériels et méthodes pour les études d'encrassement de surfaces d'échange de chaleur par du lactosérum, du lait et des fluides modèles. Annexe 3. C.R.fin Et. Interactions Lait - Matériaux solides. Contrat MIR 83 C 0165.

Daufin G., Labbé J.P., Quemerais A., Brulé G., Michel F., Roignant M., Priol M., 1987. Fouling of a heat exchange surface by whey, milk and model fluids. An analytical study. Lait, 67 (3), 339-364.

Dupeyrat M., 1983. 1. Agents de surface et émulsions. In : Galenica 5 - Les systèmes dispersés, Ed. Puisieux F., Seiller M., Technique et documentation, Lavoisier, Paris, 64-66.

Dupré A., 1869. Théorie mécanique de la chaleur, Paris, 369.

Good R.J., 1979. Contact angle and the surface free energy of solids. In : Surface and Colloid Science 1I, Ed. Good R.J. and Stromberg R.R., Plenum Press, New York and London, 1013.

Gordon K.P., Hankinson D.J., Carver C.E., 1968. Deposition of milk solids on heated surfaces. J. Dairy Sci., 51 (4), 520-526.

Guastalla J., 1952. Contribution à l'étude du mouillage. I. Introduction à l'étude tensiométrique du mouillage. J. Chim. Phys., 51, 250-261. 
Hamilton W.C., 1972. A technique for the characterization of hydrophilic solid surfaces. $J$. Colloid Interface Sci., 40 (2), 219-222.

HegG P.-O., Larsson K., 1981. Ellipsometry studies of adsorbed lipids and milk proteins on metal surfaces. In : Fundamentals and applications of surface phenomena associated with fouling and cleaning in food processing. Ed. Hallstrom B., Lund D.B., Tragardh Ch., Lund University, $250-255$.

Heughebaert J.C., Montel G., 1970. Sur l'existence d'une série de solides de composition variable correspondant au phosphate tricalcique précipité. C.R. Acad. Sci. Paris, 270, série C., 104-107.

IVARSSON B.A., JonsSON U., LUNDSTROM I., 1981. Ellipsometrical and electrochemical studies of adsorption at solid/liquid interfaces. In : Fundamentals and applications of surface phenomena associated with fouling and cleaning in food processing. Ed. Hallstrom B., Lund D.B., Tragardh Ch., Lund University, 269-278.

Ivarsson B.A., Hegg P.-O., Lundstrom I., Jonsson U., 1985. Adsorption of proteins on metal surfaces studied by ellipsometric and capacitance measurements. Colloid Surf., 13, 169-192.

Lalande M., Corrieu G., 1981. Fouling of a plate heat exchanger by milk. In : Fundamentals and applications of surface phenomena associated with fouling and cleaning in food processing. Ed. Hallstrom B., Lund D.B., Tragardh Ch., Lund University, 279-288.

lalande M., Corrieu G., Daufin G., Gallot-lavallée T., 1981. Etude des cinétiques d'encrassement et de nettoyage d'un pasteurisateur de lait à plaques. C.R. fin d'études D.G.R.S.T., 79.7.0375.

LiNG A.C., LUND D.B., 1978. Apparatus for studying fouling of heated surfaces by biological fluids. J. Food Sci., 43 (2), 390-393, 403.

Lund D.B., Bıxвy D., 1975. Fouling of heat exchange surfaces by milk. Process Biochem., 10 (9), $52-55$.

LUND D.B., SANDu C., 1981. State of the art of fouling: heat transfer surfaces. In : Fundamentals and applications of surface phenomena associated with fouling and cleaning in food processing. Ed. Hallstrom B., Lund D.B., Tragardh Ch., Lund University, 25-56.

Marriott J., 1971. Where and how to use plate heat exchangers. Chem. Eng., 78 (8), 127-134.

Nordin S., Westergren L., 1981. Practical experiences of preventing scaling by means of high finish surfaces in evaporator tubes. In : Fundamentals and applications of surface phenomena associated with fouling and cleaning in food processing. Ed. Hallstrom B., Lund D.B., Tragardh Ch., Lund University, 356-364.

NovaK L.. 1981. Fouling in plate heat exchangers and its reduction by proper design. Int. Seminar Advancement in heat exchangers; Dubrovnik, September 7-12.

Roignant M., Daufin G., Michel F., 1983. Dispositif expérimental pour étudier l'encrassement des échangeurs de chaleur alimentaires. Lait, 63,363-390.

WenNERBERG J., 1981. Observations of behavior of fouling and cleaning in industrial process. In : Fundamentals and applications of surface phenomena associated with fouling and cleaning in food processing. Ed. Hallstrom B., Lund D.B., Tragardh Ch., Lund University, 76-89.

Wilhelmy L., 1863. Ueber die Abhangigkeit der Capillaritat-constanten des Alkools von Substanz in Gestalt des benutzen festen Korper. Ann. Physik., 119, 177. 